\section{Role, timing and technique of radiotherapy in pediatric pleuropulmonary synovial sarcoma}

\author{
Corrado Spatola ${ }^{*, 1}$, Alessandra Tocco', Roberto Milazzotto', Antonio Pagana', \\ Ilenia Chillura', Roberta Bevilacqua', Carmelo Militello', Vincenzo Salamone', Luigi \\ Raffaele', Marcello Migliore² \& Giuseppe Privitera'
}

The management of pediatric thoracic synovial sarcoma remains a matter of debate in clinical oncology, especially as regard to the local control of the disease. Surgery remains the gold standard, while the role and timing of radiotherapy is still controversial. We report a 14-year-old male, who has not received proper treatment at the time of diagnosis and initial management. Intensity-modulated irradiation was performed only at relapse, as a salvage treatment and, at 10-month follow-up, the young patient was free from relapse, without significant acute and subacute toxicity. We discuss the role and timing of radiotherapy in thoracic synovial sarcoma, a disease in which the need to increase local control should be placed in the foreground.

First draft submitted: 14 July 2016; Accepted for publication: 9 September 2016; Published online: 23 September 2016

Synovial sarcoma (SS) is a rare soft-tissue sarcoma, typical of childhood, which poses serious challenges in clinical management, in particular concerning the role and timing of radiotherapy.

In this paper, we discuss a case of pediatric pleuropulmonary SS, which is a very rare localization in a site with several surrounding healthy tissues, such that the concern for secondary radiation-induced damages can lead in some circumstances to an erroneous choice to avoid a local radiotherapy.

\section{Clinical case}

A 14-year-old male, after onset of dyspnea, cough and hemoptysis, was admitted in October 2014 to the Pediatric department. Thoracic CT scan showed evidence of a voluminous neoplasia $(9.5 \mathrm{~cm}$ in maximum diameter) of the left lower lobe infiltrating the ipsilateral hilum and the costal pleura (see Figure 1, left side). A ${ }^{18} \mathrm{~F}$-fluorodeoxyglucose-PET-computed tomography $\left({ }^{18} \mathrm{~F}-\mathrm{FDG}\right.$-PET-CT) did not show distant metastases of the disease.

At another center, the option of neoadjuvant chemotherapy was not recommended to the patient, so he underwent radical surgery with a left lower lobectomy, lingulectomy and hilar-mediastinal lymphadenectomy. The histologic study diagnosed a monophasic synovial sarcoma (SYT-SSX1 fusion protein positive), grade III sec French Federation of Cancer Centers (FNCLCC), tumor necrosis, fibrosis and lymphatic invasion. Tumor infiltrated the visceral pleura and some bronchial structures with intraluminal tumor growth. There were metastases to the 1/10 left hilar lymph nodes, but surgical margins were free.

The patient was included in the EpSSG NRSTS 2005 protocol, in IRS group III, and he was subsequently subjected to adjuvant chemotherapy with ifosfamide and doxorubicin for six cycles.

\section{KEYWORDS}

- radiotherapy • sarcoma

- synovial sarcoma 

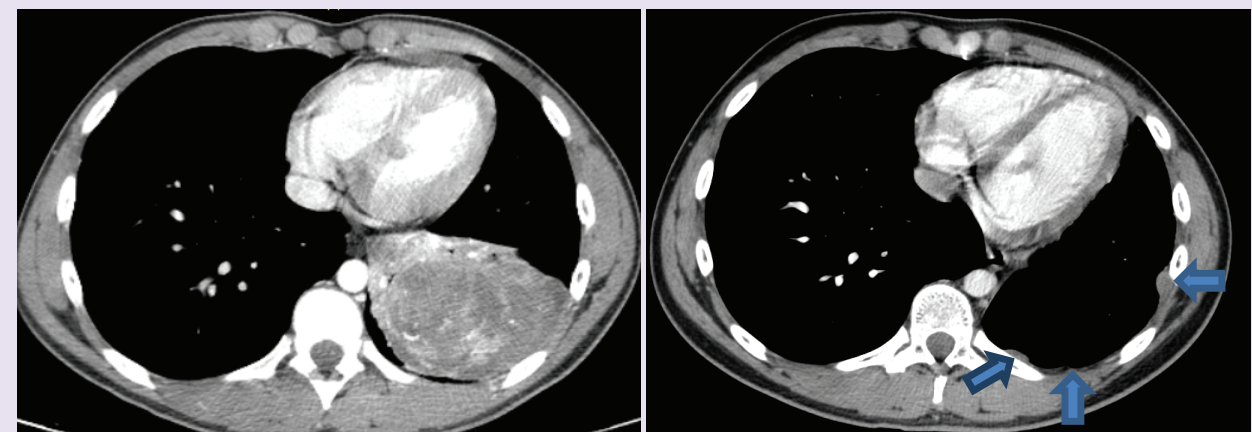

Figure 1. Axial computed tomography images showing the initial neoplasia in the left lower lobe infiltrating the hilum and the costal pleura (on the left) and the disease relapse in the left pleural cavity (on the right, the blue arrows indicate the subpleural multiple nodules, histologically proven).

Contrary to the indications of the study protocol, adjuvant radiotherapy was not recommended to the patient, because of the concerns about inducing heart or lung toxicities. We point out that the decision was taken without consulting the radiation oncologist of our center.

After a disease-free interval of only 6 months, local pluricentric nodules in the left pleural cavity were detected on CT scan (see Figure 1, right side), and histologically confirmed as SS multiple relapses.

Only at that point, the patient was referred to our Radiotherapy department and thoracic irradiation was proposed as salvage therapy. A complex intensity-modulated irradiation (IMRT) technique, concomitant to chemotherapy with ifosfamide continuous infusion, was used in order to reduce dose to the surrounding normal tissues.

The whole inferobasal pleural cavity and the ipsilateral hilum were enclosed in the clinical target volume and irradiation was carried out through eight static 'step and shoot' fields (IMRT), with a dose of 54 Gy in 30 fractions. The dose-volume histograms showed a V20 $=38 \%$ for left lung, a V20 = 12\% for the right lung and a V25 = 15\% for the heart. Thus, according to QUANTEC (quantitative analyses of normal tissue effects in the clinic), doses to the left lung and to the heart were slightly above the constraints. During the treatment period, the patient developed a grade II esophagitis, while no hematological toxicity was reported. After a short follow-up of 10 months, no local relapse or subacute toxicity occurred, as ${ }^{18}$ F-FDG-PET-CT showed at 3 and 9 months post-treatment. Obviously, a longer follow-up is needed to assess late lung/heart toxicity and the duration of local control.

\section{Discussion}

SS is a rare tumor, with less than three cases per million being diagnosed each year, it is more common among teenagers and young adults. The disease starts most commonly in the legs or arms, but it can appear in any part of the body, the thoracic location is very rare [1,2].

The data in the literature show that the tumor site should be considered when defining a risk-adapted treatment strategy for SS. In a study from the Associazione Italiana di Emato-Oncologia Pediatrica - Soft Tissue Sarcoma Committee (AIEOP-STSC) on 115 pediatric SS cases, the role of the tumor site was clearly highlighted. Patients with nonextremity SS (e.g., lung or pleura) had a 5-year overall survival of 55 versus $84 \%$ of patients with limb tumors, mainly due to local failure [3].

Surgery is considered the treatment of choice but the rate of local control is very low in local advanced disease. A multimodal approach, including surgical resection, chemotherapy and radiotherapy, has generally been suggested. The quality of surgery that patients receive and the characteristics of the disease (including tumor size, local invasiveness, histological subtype, presence of metastases and lymph node involvement) influence prognosis in SS patients. The risk of local relapse and of developing distant metastases is higher for patients with tumors that are larger than $5 \mathrm{~cm}$. Patients with the high-grade subtypes are considered to have a worse prognosis than those with other subtypes [4]. 
The case shown, as mentioned, contains some inconsistencies, especially in the initial management which occurred at other cancer center, like the surgical management as initial approach, without a neoadjuvant treatment. The patient was included in Intergroup Rhabdomyosarcoma Study (IRS) III group [5], according to the European pediatric Soft Tissue Sarcoma Study Group protocol (EpSSG NRSTS 2005) and he underwent to adjuvant chemotherapy treatment with ifosfamide-doxorubicin. There were at least three risk factors for poor prognosis and local recurrence: tumor site, tumor size and highgrade histology. All three factors are an indication for adjuvant radiotherapy but the evidence of free surgical margins and the concern for radiation-induced damages, in particular for childhood, have been a deterrent to its application in adjuvant setting [6]. We reiterate that, until that time, the patient was not referred to our Radiation Oncology department, so the decision to avoid radiotherapy was not taken in a multidisciplinary discussion.

On the other hand, clinicians have to pay attention to the prognostic importance of a local relapse. An Italian analysis on 44 relapsing cases in a series of 118 consecutive patients less than 21 years of age with nonmetastatic synovial sarcoma, showed a particularly poor outcome for relapsing tumors, with an overall survival of $29.7 \%$ at 5 years for SS patients whose tumors relapse [7].

According to the EpSSG protocol, surgery could be considered the unique local treatment for group I patients, while postoperative radiotherapy is required for IRS group II patients [8]. IRS group III patients are at high risk of local relapse, with an incidence of local failure of about $44 \%$ [9], and radiotherapy is almost always carried out. There is not a clear consensus on the timing of it (pre-operative vs postoperative), and the mainstay of treatment is to obtain a secondary complete resection (when applicable).

In our case, it was not clear to which prognostic group the patient belonged, because the tumor diameter was greater than $5 \mathrm{~cm}$ and poorly differentiated, but the first surgery was R0. Hence, despite him being included in IRS Group III for N1 disease, there was no need for a secondary surgery. Therefore, radiotherapy would have a role as an adjuvant treatment, as for IRS group I or II patients. Literature reports a 5 -year local relapse-free survival of $78 \%$ in
IRS group I patients (R0 at surgery) treated with radiotherapy and $67 \%$ in those treated without postoperative radiotherapy. The benefit of adjuvant radiotherapy is more evident in IRS group II patients (R1 at surgery), with a local relapse-free survival rate at 5 years of $75.7 \%$ in patients administered radiotherapy and $55.6 \%$ in patients who did not receive radiotherapy [10].

However, the patient was referred to us only at the time of pleural recurrence, when only a salvage treatment could be done. We decided to perform a concomitant radiochemotherapy treatment, with thoracic IMRT and ifosfamide continuous infusion. It was well tolerated by the patient, with the occurrence of a grade II esophagitis.

To our knowledge, the literature does not report any experience about the use of intensitymodulated radiotherapy for the treatment of pleuropulmonary SS, neither in adjuvant setting nor in relapsing tumors. Nevertheless, there is increasing interest in its use for the treatment of various types of thoracic tumors, thanks to the ability of IMRT to reduce radiation dose delivered to healthy tissues.

\section{Conclusion}

The optimal management of SS remains to be determined, in particular for rare localizations. Surgery is the mainstay of treatment, both as initial approach and as a second option. The role and timing of radiotherapy and chemotherapy are a matter of debate. Radiotherapy has a role in improving local control but it is often avoided when a $\mathrm{R} 0$ resection is obtained. In our opinion, all prognostic factors (including tumor size, site of localization, tumor grading) should be considered when a treatment strategy must is programmed. The wide distribution and the increasing indications pointing to IMRT should encourage clinicians to avoid the underutilization of radiotherapy, in particular when local recurrence significantly influences overall survival. Moreover, the perspective of tailored treatment strategy should always be discussed in a multidisciplinary setting.

The case presented unfortunately describes a not very rare reality in clinical practice. Radiation therapy is often avoided or postponed in rare thoracic tumors, with no real clinical grounds, but only for the supposed risk of serious side effects and, above all, without the 
radiation oncologist is involved in decision making. However, the case needs a longer follow-up to assess the duration of local control and the occurrence of late toxicities.

Financial \& competing interests disclosure

The authors have no relevant affliations or financial involvement with any organization or entity with a financial interest in or financial conflict with the subject matter or materials discussed in the manuscript. This includes employment, consultancies, honoraria, stock ownership or options, expert testimony, grants or patents received or pending, or royalties.
No writing assistance was utilized in the production of this manuscript.

Informed consent disclosure

The authors state that they have obtained verbal and written informed consent from the patient/patients for the inclusion of their medical and treatment history within this case report.

\section{Open access}

This work is licensed under the Creative Commons Attribution-NonCommercial 4.0 Unported License. To view a copy of this license, visit http://creativecommons.org/ licenses/by-nc-nd/4.0/

\section{EXECUTIVE SUMMARY}

- Synovial sarcoma (SS) is a rare soft-tissue sarcoma, typical of childhood.

- Its management remains a matter of debate in clinical oncology, especially as regard the local control of the disease. Surgery remains the gold standard, while the role and timing of radiotherapy is still controversial.

- In this paper, the authors discuss a case of a 14-year-old male with pleuropulmonary SS, which is a very rare localization in a site with several surrounding healthy tissues, such that the concern for secondary radiation-induced damages can lead in some circumstances to an erroneous choice to avoid a local radiotherapy.

- The patient did not receive proper treatment at the time of diagnosis and initial management: at another center where the option of a neoadjuvant chemotherapy was not recommended to the patient, so he underwent to a radical surgery, with a diagnosis of monophasic SS (SYT-SSX1 positive).

- Adjuvant chemotherapy was performed, according to the European Pediatric Soft Tissue Sarcoma Study Group prospective trial (EDSSG NRSTS 2005) protocol, but adjuvant radiotherapy was not recommended, due to the concern for radiation-induced side effects, thus resulting in an early relapse, after 6 months.

- The authors point out that they do not share clinical decisions until the time of the relapse, when patient was finally referred to the radiotherapy department.

- A salvage treatment was performed at that time. A complex intensity-modulated irradiation (IMRT) technique, concomitant to chemotherapy with ifosfamide continuous infusion, was used in order to reduce dose to the surrounding normal tissues.

- The whole inferobasal pleural cavity and the ipsilateral hilum were enclosed in the clinical target volume and irradiation was carried out through eight static 'step and shoot' fields (IMRT), with a dose of 54 Gy in 30 fractions.

- During the treatment period, the patient developed a grade II esophagitis, while no hematological toxicity was reported. After a short follow-up of 10 months, no local relapse or subacute toxicity occurred, as ${ }^{18} \mathrm{~F}$-FDG-PET-CT showed at 3- and 9-month post-treatment.

- A longer follow-up is needed to assess late lung/heart toxicity and the duration of local control.

- The authors discuss the role and timing of radiotherapy in thoracic SS, a disease in which the need to increase local control should be placed in the foreground.

- The literature report a 5-year local relapse-free survival of 78\% in Intergroup Rhabdomyosarcoma Study group I patients (R0 at surgery) treated with radiotherapy and $67 \%$ in those treated without postoperative radiotherapy. The benefit of adjuvant radiotherapy is more evident in Intergroup Rhabdomyosarcoma Study group II patients (R1 at surgery), with a local relapse-free survival rate at 5 years of $75.7 \%$ in patients administered radiotherapy and $55.6 \%$ in patients who did not receive radiotherapy.

- The literature does not report any experience about the use of IMRT for the treatment of pleuropulmonary SS, neither in adjuvant setting nor in relapsing tumors. Nevertheless, there is increasing interest in its use for the treatment of various types of thoracic tumors, thanks to the ability of IMRT to reduce radiation dose delivered to healthy tissues. 


\section{Bibliography}

Papers of special note have been highlighted as:

- of interest; $\bullet \bullet$ of considerable interest

1 Sultan I, Rodriguez-Galindo C, Saab R et al. Comparing children and adults with synovial sarcoma in the Surveillance, Epidemiology and End Results Program, 1983 to 2005: an analysis of 1268 patients. Cancer 115, 3537-3547 (2009).

2 WHO Classification of Tumours. Pathology and Genetics. Tumours of Soft Tissue and Bone. Fletcher CDM, Unni KK, Mertens F (Eds). IARC Press, Lyon, France (2002).

3 Ferrari A, Bisogno G, Alaggio R et al. Synovial sarcoma of children and adolescents: the prognosticrole of axial sites. Eur. J. Cancer 44(9), 1202-1209 (2008).

- Regarding the general management of synovial sarcoma (SS), particularly its treatment strategy and the timing of interventions.
4 Spillane AJ, A'Hern R, Judson IR et al. Synovial sarcoma: a clinic-pathologic, staging, and prognostic assessment. J. Clin. Oncol. 18, 3794-3803 (2000).

5 Maurer HM, Beltangady M, Gehan EA et al. The Intergroup Rhabdomyosarcoma Study I: a final report. Cancer 61, 209-220 (1988).

6 Pappo AS, Fontanesi J, Luo X et al. Synovial sarcoma in children and adolescents: the St. Jude Children's Research Hospital experience. J. Clin. Oncol. 12, 2360-2366 (1994).

7 Ferrari A, Salvo GL, Dall'igna P et al. Salvage rates and prognostic factors after relapse in children and adolescents with initially localized synovial sarcoma. Eur. J. Cancer 48(18), 3448-3455 (2012).

- Regarding the general management of SS, particularly its treatment strategy and the timing of interventions.

8 Ferrari A, De Salvo GL, Brennan B et al. Synovial sarcoma in children and adolescents: the European Pediatric Soft Tissue Sarcoma
Study Group prospective trial (EpSSG NRSTS 2005). Ann. Oncol. 26(3), 567-572 (2015).

- $\quad$ Regarding the study protocol EpSSG NRSTS 2005.

9 Spunt SL, Ashley Hill D, Motosue AM et al. Clinical features and outcome of initially unresected nonmetastatic pediatric non rhabdomyosarcoma soft tissue sarcoma. J. Clin. Oncol. 20, 3225-3235 (2002).

- Regarding the general management of SS, particularly its treatment strategy and the timing of interventions.

10 Ferrari A, Casanova M, Collini P et al. Adult-type soft tissue sarcomas in pediatric-age patients: experience at the Istituto Nazionale Tumori in Milan. J. Clin. Oncol. 23(18), 4021-4030 (2005).

- Regarding the general management of SS, particularly its treatment strategy and the timing of interventions. 